\title{
The role of serum biomarkers in the diagnosis and prognosis of oral cancer: A systematic review
}

\author{
Ana Fernández-Olavarría ${ }^{1}$, Regina Mosquera-Pérez ${ }^{1}$, Rosa-María Díaz-Sánchez ${ }^{1}$, Maria-Angeles Serrera- \\ Figallo $^{2}$, José-Luis Gutiérrez-Pérez ${ }^{3}$, Daniel Torres-Lagares ${ }^{4}$
}

\author{
${ }^{1}$ DDS.School of Dentistry. University of Seville \\ ${ }^{2} \mathrm{PhD}$, DDS. School of Dentistry. University of Seville \\ ${ }^{3}$ DMD. Professor of Oral Surgery. Chairman of Oral Surgery. Department of Stomatology. University of Seville \\ ${ }^{4} \mathrm{PhD}$, DDS, MSc (Oral Surgery). Proffesor of Oral Surgery. Department of Stomatology. University of Seville
}

Correspondence:

School of Dentistry of Seville

C/ Avicena s/n 41009

Seville. Spain

danieltl@us.es

Received: $13 / 08 / 2015$

Accepted: 30/11/2015

\begin{abstract}
Fernández-Olavarría A, Mosquera-Pérez R, Díaz-Sánchez RM, SerreraFigallo MA, Gutiérrez-Pérez JL, Torres-Lagares D. The role of serum biomarkers in the diagnosis and prognosis of oral cancer: A systematic review. J Clin Exp Dent. 2016;8(2):e184-93.

http://www.medicinaoral.com/odo/volumenes/v8i2/jcedv8i2p184.pdf
\end{abstract}

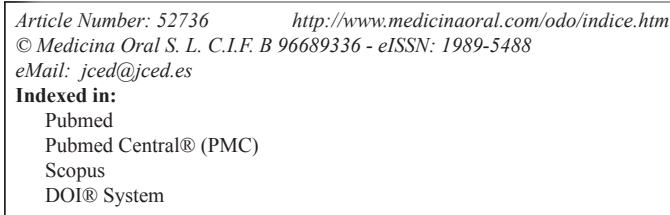

\begin{abstract}
Introduction: Oral cancer is one of the causes of major morbidity and mortality in the world although incidence varies in the different geographical locations and races. Advances in molecular biology and cancer research have allowed elucidating serum biomarkers to improve diagnostic methods. The aim of this article systematic review is to highlight the utility and clinical value of serum biomarkers in the diagnosis and prognosis of oral cancer.

Material and Methods: A systematic literature review using PubMed (MEDLINE databases) revealed a total of 140 articles related to this topic. Of those articles, 29 were included in the final review. We included articles published in English in the last five years, developed in human as cases and controls studies, retrospective or prospective studies and specific studies that analyzed a certain biomarker in serum.

Results: All of the studies include in this systematic review found significant differences in patients. Of those articles included, 2 used biomarkers to determinate cancerous phenotype, 11 mentioned their results were associated with worse prognosis and overall survival, 4 correlated biomarker concentration to clinical stages, 4 concluded it could be a helpful in diagnosis and 8 studies did not find a clear utility of the analysed biomarker. Due to differences in the presentation of data, meta-analysis was not possible.

Conclusions: Biomarker use for diagnosis and prognosis is supported by clinical and scientific evidence is relevant. Nevertheless, after selecting a certain biomarker, monitoring protocols should be established in oral and maxillofacial surgeons teams so as we have a correct understanding of biological values.
\end{abstract}

Key words: Serum biomarkers, oral cancer, diagnosis, prognosis.

\section{Introduction}

Oral cancer is the sixth most common malignancy worldwide. Aproximately, $90 \%$ of cancer located in the oral cavity are oral squamous cell carcinoma (OSCC)
(1). Most oral cancers are superficial and easily detected, but deeply located tumors may not be noted until they have grown large and reached an advanced stage. This malignant neoplasm occurs most commonly in the pos- 
teriorlateral border and ventral surfaces of the tongue. The second most common location is the floor of the mouth (2). Due their aggressiveness, oral cancer invades surrounding organs and causes regional or distant metastases (3). The overall survival rate for oral cancer is considerably lower than that of other cancers due to metastasis and recurrence (4).

Epidemiological studies showed variable incidences depending on the region. Incidence is particularly high in India, Brazil, Pakistan and France. Tobacco (particularly chewing) and alcohol have been large demonstrated as risk factors in the development of oral cancer (5). Additionally, these risk factors have been showed a synergist effect when they have been combined (6).

The determination of serum biomarkers is accepted as a valuable tool for diagnosis, finding therapeutic targets and prognosis in different kind of tumors (7). Literature has been showed overexpression in serum of some proteins (8), p53 antibody (9), and VEGF (8) as an indicator of oral cancer. Several biomarkers have been proposed, but they are sometimes variable with race, lifestyle, and carcinogen exposure. The global knowledge of all of them would lead to the improvement of diagnostic and prognosis methods of tumor recurrence and metastasis to assess changes in oral lesions (3).

-Serum biomarkers

Serum biomarkers are defined as substances changing quantitatively in the serum during tumor development. Classically, a marker is synthesized by the tumor and released into circulation or expressed at the cell surface in large quantity by malignant cells (10). These markers can been used in the prognosis of tumor recurrence or metastasis (11) because the development of the malignant tumor changing their concentrations (7). The tumor marker/substance can be classified as tumor specific and tumor associated. Tumor specific substance are considered as a direct result of oncogenesis, while tumor associated marker are various proteins, enzymes, hormones and immunoglobulins which occur in the blood and are mediated by the tumor itself or by the influence of the tumor on the involved tissues (12). Repeating test of serum biomarker allows following treatment and assessing response to treatment, monitoring tumor progression and metastasis (13). However, there are not yet unified parameters to determinate which biomarker would be useful for oral cancer.

The main focus of this systematic review is to analyze the utility of serum biomarkers in the diagnosis and prognosis of oral cancer.

\section{Material and Methods}

-Search Strategy and Selection criteria

A systematic, computerized database search was conducted using the National Center for Biotechnology Information (NCBI) to search MEDLINE (Pub- med). The search was conducted using the following MeSHterms:"mouth neoplasms" AND marker AND (serum OR blood) [Mesh].

For the initial selection, article titles and/or abstracts were analyzed and the following inclusion criteria were observed: studies published in English in the last five years; studies of human beings; specific studies that analyzed a certain biomarker in serum; and study type: cases and controls studies, prospective and/or retrospective clinical studies. The exclusion criteria were: studies which do not mention the measurement method, studies that analyses markers in saliva.

Following initial selection, we read the previously selected articles fully, applying the selection criteria (Fig. 1) to determine final inclusion or exclusion from the study.

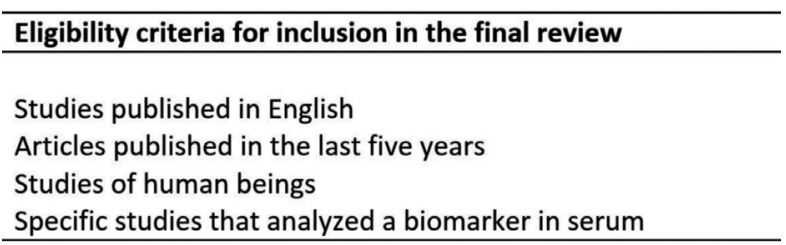

Fig. 1. Eligibility criteria for inclusion in the final review.

\section{-Quality rating}

A methodological quality rating was performed according to the PRISMA statement criteria in order to verify the strength of scientific evidence in clinicaldecisionmaking. The classification of the risk of bias potential for each study was based on the criteria adopted by Clementini et al. (14) described as follows: random selection of the sample; definition of inclusion/exclusion criteria; follow-up reports; validated measurements; statistical analysis. A study that included all the criteria mentioned above was classified as having a low risk of bias; astudy that did not include one of these criteria was classified as having a moderate risk of bias; when two or more criteria were missing, the study was assigned a high risk of bias (Table 1).

\section{Results}

The electronic database search was performed on December, 2013 and yielded 130 results. Seventy articles were identified as relevant after reading the title and/or abstract. The full text of these 70 papers was evaluated according to the selection criteria in table 1 . Of these 70 articles, six did not fulfill one or more selection criteria and were excluded. Twenty-seven articles were included in the final review. A flowchart of the selection and evaluation processes is shown in figure 2 .

Of the articles included in the final review, twenty-two were cases/controls studies, one was a cohort study, two were prospective, and two were retrospective. The sam- 
Table 1. Quality assessment of the prospective and retrospective studies included.

\begin{tabular}{|c|c|c|c|c|c|c|c|}
\hline Year & Author & $\begin{array}{l}\text { Random } \\
\text { selection in } \\
\text { population }\end{array}$ & $\begin{array}{c}\text { Defined } \\
\text { inslusion/exclusion } \\
\text { criteria }\end{array}$ & $\begin{array}{c}\text { Report loss } \\
\text { to } \\
\text { follow-up }\end{array}$ & $\begin{array}{c}\text { Validated } \\
\text { measurements }\end{array}$ & $\begin{array}{c}\text { Statistical } \\
\text { analysis }\end{array}$ & $\begin{array}{c}\text { Estimated } \\
\text { potential } \\
\text { risk of } \\
\text { bias }\end{array}$ \\
\hline 2014 & Pajkumar, N & Cases/Controls & Yes & No & Yes & Yes & Moderate \\
\hline 2013 & Xia O-Hong, G & Cases/Controls & Yes & No & Yes & Yes & Moderate \\
\hline 2013 & Tsai, YD & Prospective & Yes & Yes & No & Yes & Moderate \\
\hline 2013 & Chang, KP & Cases/Controls & Yes & No & Yes & Yes & Moderate \\
\hline 2013 & Chang, KP & Cohort study & Yes & No & Yes & Yes & Moderate \\
\hline 2013 & Ratajczak-wana, W & Cases/Controls & Yes & No & No & Yes & High \\
\hline 2012 & Huang,SF & Retrospective & Yes & Yes & Yes & Yes & Low \\
\hline 2012 & Schiegnitz, E & Cases/Controls & Yes & No & Yes & Yes & Moderate \\
\hline 2012 & Brailo, V & Cases/Controls & Yes & No & Yes & Yes & Moderate \\
\hline 2012 & Brailo, V & Cases/Controls & Yes & No & Yes & Yes & Moderate \\
\hline 2012 & Tadbir, AA & Cases/Controls & Yes & No & Yes & Yes & Moderate \\
\hline 2012 & Cheng, SJ & Cases/Controls & Yes & Yes & Yes & Yes & Low \\
\hline 2011 & Chang,KP & Cases/Controls & Yes & Yes & Yes & Yes & Low \\
\hline 2011 & Cordella, C & Retrospective & Yes & Yes & Yes & Yes & Low \\
\hline 2011 & Prabhu, K & Cases/Controls & Yes & Yes & Yes & Yes & Low \\
\hline 2011 & Joshi, M & Cases/Controls & Yes & No & Yes & Yes & Moderate \\
\hline 2011 & $\mathrm{Li}, \mathrm{C}$ & Cases/Controls & Yes & Yes & Yes & Yes & Low \\
\hline 2011 & Sawant & Cases/Controls & Yes & Yes & Yes & Yes & Low \\
\hline 2011 & Nayak, S & Cases/Controls & Yes & No & Yes & Yes & Moderate \\
\hline 2010 & Batista Faria, P & Cases/Controls & Yes & Yes & No & Yes & Moderate \\
\hline 2010 & Tu, HF & Cases/Controls & Yes & No & Yes & Yes & Moderate \\
\hline 2010 & Tamaki, S & Cases/Controls & Yes & Yes & Yes & Yes & Low \\
\hline 2010 & Feng, XY & Cases/Controls & Yes & Yes & Yes & Yes & Low \\
\hline 2010 & Harshkant, P & Cases/Controls & No & Yes & Yes & Yes & Moderate \\
\hline 2010 & Friedrich & Prospective & No & Yes & No & Yes & High \\
\hline 2009 & Khandavilli, SD & Prospective & Yes & Yes & Yes & Yes & Low \\
\hline 2009 & Liu, CJ & Cases/Controls & Yes & Yes & Yes & Yes & Low \\
\hline
\end{tabular}

ple size of each cases/controls study ranged from 27 to 237 for patients and 14 to 112 for healthy controls (Table 2).

The most used measurement method was ELISA (enzyme-linked immunosorbent assay) in 17 studies, whereas other authors used Immunohistochemistry, Western Blott, flowcytometric analysis, IRMA (immunoradiometric assay), Resorcinol Reagent Method, Cystein Reagent method, particle enhanced turbimetric assay technique and PCR (Polymerase Chain Reaction) (Table 2). The twenty-six articles included in the final review reported twenty-two different biomarkers used in diagnosis and/or prognosis of oral cancer. Of those, four were specific tumor markers, direct result of oncogenesis, and twelve were associated tumor markers, substances mediated by the tumor (Table 3).

Regarding the quality assessment, twelve studies achieved low risk of bias. Twelve studies were determined moderate risk and two studies were assigned to have high risk of bias (Table 1).

\section{Discussion}

Biomarkers have been wide accepted in other disciplines but there is no consensus for their use in oral malignan- cies. Despite recent advances in surgical, radiotherapy, and chemotherapy treatment protocols, the survival of patients with OSCC still lacks significant improvement. This unsatisfactory treatment may be explained by the fact that OSCCs frequently present with extensive local invasion and advanced stages $(15,16)$. That makes necessary the development of new tools for the diagnosis and prognosis.

Tumor growth, invasion and metastasis are multiple step processes in which many genes and molecules are involved. The molecular biology of OSCC is complex and OSCC develops from the dysfunction of several interrelated pathways (17).

Our systematic review shows how several authors in the last years have looked for the best marker for diagnose oral cancer at earlier stages, establish the prognosis and increase the survival of patients with this disease.

-Adiponectine

Adiponectin is an adipokine produced predominantly by adipocytes that circulates abundantly in plasma and functions as an anti-diabetic, anti-atherogenic, anti-inflammatory and anti-angiogenic hormone (18).

In their study Guo et al. (19) showed that serum adiponectin level was reduced in tongue squamous cell car- 


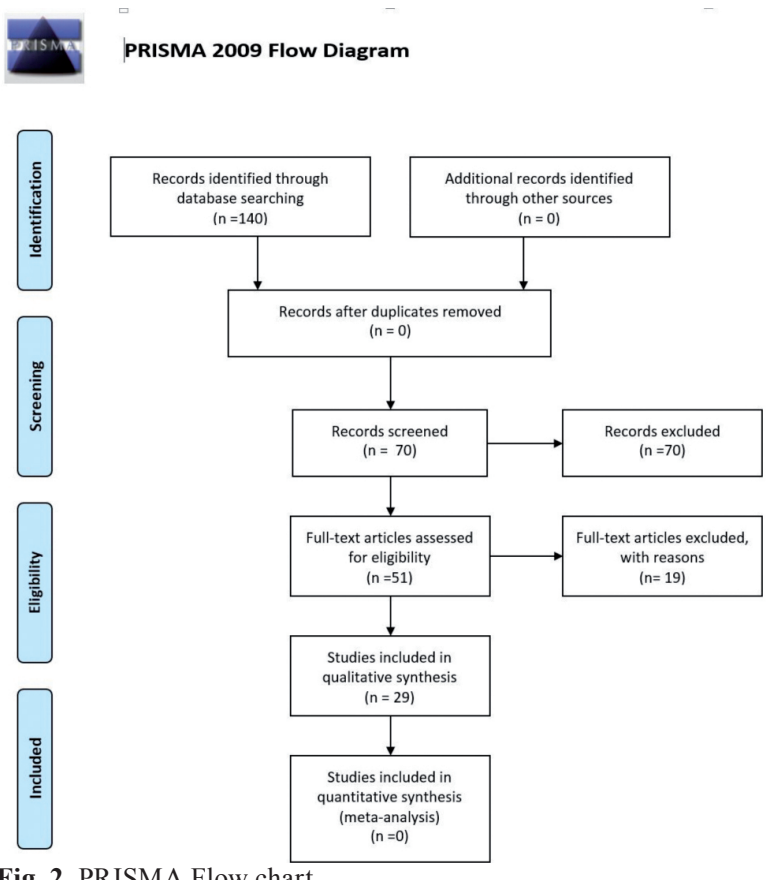

Fig. 2. PRISMA Flow chart.

cinoma (TSCC), and inversely associated with histological grade and lymphnode metastasis of TSCC. They suggested that hypoadiponectinemia is correlated with histopathologic features of TSCC, and could be a new biomarker of aggressive phenotype in TSCC. But they still reckon the underlying mechanisms of adiponectin in potential cancer suppression are not fully elucidated. -Annexin A1 mRNA

Annexin A1 an anti-inflammatory and calcium-dependent protein of the superfamily of annexins, may have important regulatory roles in tumor development and progression (20). The Annexin A1 gene expression was investigated by Faria et al. (21), in peripheral blood samples of patients with oral squamous cell carcinoma and control subjects and Annexin A1 mRNA was expressed in all of them. Comparative analysis of OSCC blood patients showed significantly lower Annexin A1 expression when compared to blood sample of control individuals. However, there were no significant differences between patients' subgroups in relation to smoking, drinking, recurrence, TNM staging histopathological grading or therapies. This present study revealed the Annexin mRNA as new possible transcript biomarker for early detection of OSCC in the peripheral blood of patients.

-Cyclin D1

Xiao-yu Feng et al. (22) measured the level of some biomarkers (SCCA, Cyfra 21-1, epidermal growth factor receptor (EGFR) and Cyclin D1) in an attempt to determine the usefulness of their combined determination in the diagnosis of OSCC. They concluded that Cyclin D1, the product of the CCND1 gene located on chromosome 11q13, had the highest diagnostic specificity. Moreover the combined detection of EGFR and Cyclin D1 had the highest sensitivity, specificity and accuracy.

A previous study (23), demonstrates that Cyclin D1expression was significantly associated with the presence of occult lymph node metastases. These data suggest that the immunohistochemical analysis of Cyclin D1 expression in diagnostic biopsy samples may be an additional tool for selecting patients to be treated with elective neck dissection. -C-Reactive Protein (CRP)

CRP is a functional analogue to immunoglobulin $\mathrm{G}$, which synthesis by pro-inflammatory cytokines. An increase in the value of CRP has been demonstrated in patients with inflammatory disease and various cancers. In a recent study, Khandavilli et al. (24), investigated the relationship between preoperative serum CRP levels, tumor size, stage and survival for oral cancer patients. They found that two years survival rates in patients with preoperative elevation of serum CRP, more than $5 \mathrm{mg} / \mathrm{L}$, was significantly less favorable (44\%) than that in patients without serum CRP elevation (90\%).It demonstrated the link between raised CRP and malignant potential of oral SCC, concluding that it could be used as an independent prognostic indicator for patients with oral SCC treated by primary surgery.

-Decoy receptor 3 (DcR3)

DcR3 functions as a death decoy inhibiting apoptosis mediated by the tumor necrosis factor receptor family. Frequently, gene amplification of DcR3 has been detected in various malignant tumors. Tu et al. (25) analyzed serum DcR3 level by an enzyme-linked immunosorbent assay (ELISA), quantitativepolymerase chain reaction (Q-PCR) and immunochemistry. They found that elevated serum DcR3 $(>284 \mathrm{pg} / \mathrm{ml})$ was associated with nodal metastasis and worse prognosis, concluding that serum DcR3 level is an independent prognostic factor of OSCC and also a predictor for neck nodal metastasis.

-Growth-differentiation factor (GDF 15)

Growth-differentiation factor 15 (GDF 15) is a member of the transforming growth factor-b (TGF-b) superfamily, involved in tumor pathogenesis and its expression is increased in many types of cancers (26). Schiegnitz et al. (27) reported for the first time, in vivo, enhanced serum GDF 15 levels in patients with OSCC and provided evidence demonstrating a significant relationship between serum GDF 15 levels and prognosis of the patients. However, they concluded the role of GDF 15 in cancer pathophysiology is not clear yet. The diagnostic utility of GDF 15 could be improved by combining GDF 15 with other serum markers.

-Hemoglobine $(\mathrm{Hb})$

Low $\mathrm{Hb}$ levels are indeed associated with poor tumor oxygenation and increasing $\mathrm{Hb}$ concentrations are correlated with higher pO2 levels and lower hypoxic tissue fractions. In a retrospective study, Cordella et al. (28) 
Table 2. Summarize evidence on selected papers.

\begin{tabular}{|c|c|c|c|c|c|}
\hline MARCADOR & ESTUDIO & DISEÑO & MEDICIÓN & VALOR BIOLÓGICO & RELEVANCIA \\
\hline $\begin{array}{l}\text { Adiponectine } \\
\text { (Xia O-Hong, G; 2013) }\end{array}$ & Cases/Controls & 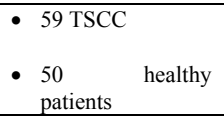 & $\begin{array}{l}\text { Immunohisto- } \\
\text { chemistry } \\
\text { Western Blot }\end{array}$ & $\begin{array}{l}\text { - } \text { TSCC }=5.0 \pm 2.4 \mu \mathrm{g} / \mathrm{ml} \\
\text { - Controls }= \\
\\
8.4 \pm 3.5 \mu \mathrm{g} / \mathrm{ml}\end{array}$ & $\begin{array}{l}\text { Hypoadiponectinemia predict } \\
\text { aggresive phenotype } \\
\text { (inverselyassociated) }\end{array}$ \\
\hline $\begin{array}{l}\text { Annexin A1 mRNA } \\
\text { (Batista Faria, P; 2010) }\end{array}$ & Cases/Controls & $\begin{array}{l}\text { - } 27 \text { OSCC } \\
\text { - } 25 \quad \text { patients }\end{array}$ & Q.R.time PCR & Lowerexpression & $\begin{array}{l}\text { Decrease associated with } \\
\text { cancerous phenotype } \\
\text { (Tumor suppressor gene) }\end{array}$ \\
\hline $\begin{array}{l}\text { CRP + SCC-Ag } \\
\text { (Huang,SF ; 2012) }\end{array}$ & $\begin{array}{l}\text { Retrospective } \\
\text { (preoperative serum) }\end{array}$ & - $142 \mathrm{OSCC}$ & ELISA & $\begin{array}{l}\text { - } \text { Cut-off SCC-Ag } \geq 2.0 \\
\text { ng/ml } \\
\text { - } \text { Cut-off CRP } \geq 5.0 \\
\text { ng/ml }\end{array}$ & Indicates overall survival \\
\hline CRP (Khandavilli,SD; 2009) & Prospective & $\begin{array}{l}\bullet \quad 60 \text { OSCC } \\
\text { Preoperative serum }\end{array}$ & $\begin{array}{l}\text { Particle } \\
\text { enhanced } \\
\text { turbimetric } \\
\text { immunoassayte } \\
\text { chnique }\end{array}$ & $\begin{array}{l}\text { - Ranged }=0.1-89.3 \\
\mathrm{mg} / \mathrm{L} \\
\text { - } 0-5 \mathrm{mg} / \mathrm{L}=\text { normal } \\
\text { - }>5 \mathrm{mg} / \mathrm{L}=\text { raised } \\
\text { (T3,T4) }\end{array}$ & Indicates overall survival \\
\hline $\begin{array}{l}\text { Citokine markers (Chang,KP; } \\
\text { 2011) }\end{array}$ & $\begin{array}{l}\text { Cases/Controls } \\
\text { proteins) }\end{array}$ & $\begin{array}{l}\text { - } 111 \text { OSCC } \\
\text { - } 112 \text { healthy } \\
\text { patients } \\
\text { - } 107 \text { premalignant } \\
\text { lesions }\end{array}$ & ELISA & $\begin{array}{l}\text { - OSCC } 12 \text { proteins } \\
\text { dysregulated }\end{array}$ & $\begin{array}{l}\text { VEGF }>4.87 \mathrm{pg} / \mathrm{ml}=\text { worse } \\
\text { prognosis OSCC }\end{array}$ \\
\hline $\begin{array}{l}\text { CXCL-9 } \\
\text { (Chang, KP; 2013) }\end{array}$ & Cases/Controls & $\begin{array}{l}\text { - } 181 \text { OSCC } \\
\text { - } 231 \quad \text { healthy } \\
\text { patients }\end{array}$ & ELISA & - CXCL-9 > $209 \mathrm{pg} / \mathrm{ml}$ & Worse prognosis \\
\hline $\begin{array}{l}\text { DCR3 } \\
\text { (Tu, HF; 2010) }\end{array}$ & $\begin{array}{l}\text { Prospective } \\
\text { (preoperative DCR3 } \\
\text { level) }\end{array}$ & - 148 OSCC & $\begin{array}{l}\text { ELISA } \\
\text { QPCR } \\
\text { Immunohistoch } \\
\text { emistry }\end{array}$ & $\begin{array}{l}\text { Follow-up } 23 \pm 11.8 \\
\text { months } \\
>284 \mathrm{pg} / \mathrm{ml}=\text { worse } \\
\text { prognosis } \\
\text { (nodal metastasis) }\end{array}$ & Predictor of survival \\
\hline $\begin{array}{l}\text { GDF } 15 \\
\text { (Schiegnitz, E; 2012) }\end{array}$ & Cases/Controls & $\begin{array}{l}\text { - } 64 \text { OSCC } \\
\text { - 30healthy patients }\end{array}$ & ELISA & $\begin{array}{l}\text { - OSCC preoperative = } \\
1545 \pm 774 \mathrm{pg} / \mathrm{ml} \\
\text { - OSCC postoperative= } \\
953 \pm 438 \mathrm{pg} / \mathrm{ml}\end{array}$ & $\begin{array}{l}\text { GDF15 serum level }<875 \mathrm{pg} / \mathrm{ml} \\
\text { =higher survival }\end{array}$ \\
\hline $\begin{array}{l}\text { Preoperative Hemoglobine } \\
\text { (Cordella, C; 2011) }\end{array}$ & $\begin{array}{l}\text { Retrospective } \\
\text { (follow-up } 12 \text { months) }\end{array}$ & $287 \mathrm{OSCC}$ & $\mathrm{Hb}$ & $\begin{array}{l}71,4 \% \text { normal } \mathrm{Hb} 18.5 \% \\
\text { mild anemia } \\
10.1 \% \text { severe anemia }\end{array}$ & $\begin{array}{l}\text { Anemia significant for the } \\
\text { development of lymph node } \\
\text { metastasis } \\
<11 \mathrm{~g} / \text { dl poor prognosis }\end{array}$ \\
\hline $\begin{array}{l}\text { TNF } \alpha \\
\text { (Brailo, V; 2012) }\end{array}$ & Cases/Controls & $\begin{array}{l}\text { - } 28 \text { OSCC } \\
\text { - } 29 \text { leucoplakia } \\
\text { - } 31 \text { healthy patients }\end{array}$ & ELISA & $\begin{array}{l}\text { - } \text { OSCC }=5 \pm 2.51 \mathrm{pg} / \mathrm{ml} \\
\text { - Leucoplakia }=6 \pm 1.06 \\
\mathrm{pg} / \mathrm{ml} \\
\text { - } \text { Controls }=8 \pm 1.34 \mathrm{pg} / \mathrm{m} \\
\\
1(\mathrm{p}=0.038)\end{array}$ & $\mathrm{TNF} \alpha>$ in control serum \\
\hline IL6 (Brailo, V; 2012) & Cases/Controls & $\begin{array}{l}\text { - } 28 \text { OSCC } \\
\text { - } 29 \text { leucoplakia } \\
\text { - } 31 \text { healthy patients }\end{array}$ & ELISA & $\begin{array}{l}\text { - OSCC }=3 \pm 0.58 \mathrm{pg} / \mathrm{ml} \\
\text { - Leucoplakia }=4 \pm 1.22 \\
\text { pg/ml } \\
\text { - Controls }=3 \pm 23 \mathrm{pg} / \mathrm{Iml} \\
(\mathrm{p}=0.989)\end{array}$ & \\
\hline IL6 (Chang, KP; 2013) & Cohort study & $\begin{array}{l}\text { - } 237 \text { OSCC } \\
\text { - } 104 \text { premalignant } \\
\text { lesions } \\
\text { - } 125 \quad \text { healthy } \\
\text { patients }\end{array}$ & ELISA & $\begin{array}{l}\text { - } \mathrm{T} 1=0.0 \mathrm{pg} / \mathrm{ml} \\
\text { - } \mathrm{T} 2=0.0 \mathrm{pg} / \mathrm{ml} \\
\text { - } \mathrm{T} 3=1.3 \mathrm{pg} / \mathrm{ml} \\
\text { - } \mathrm{T} 4=5.0 \mathrm{pg} / \mathrm{ml} \\
(\mathrm{p}=1.35)\end{array}$ & $\begin{array}{l}\text { Independent prognosis factor for } \\
\text { overall survival }\end{array}$ \\
\hline $\begin{array}{l}\text { MiCB } \\
\text { (Tamaki, S; 2010) }\end{array}$ & Cases/Controls & $\begin{array}{l}\text { - } 60 \text { OSCC } \\
\text { - 50healthy patients }\end{array}$ & ELISA & $\begin{array}{l}\text { - Controls }=21.2 \pm 16.1 \\
\mathrm{pg} / \mathrm{ml} \\
\text { - } \mathrm{T} 1= \\
12.4 \pm 16.2 \mathrm{pg} / \mathrm{ml} 21.7 \pm \\
10.5 \mathrm{pg} / \mathrm{ml} \\
\text { - } \mathrm{T} 2= \\
\text { - } \mathrm{T} 3=30.1 \pm 17.2 \mathrm{pg} / \mathrm{ml} \\
\text { - } \mathrm{T} 4=37.8 \pm 10.1 \mathrm{pg} / \mathrm{ml}\end{array}$ & $\begin{array}{l}\text { sMICBlevels } \\
\text { significantly increased in stage IV } \\
\text { OSCC. } \\
\text { Associated with decreased } \\
\text { survival rates i }\end{array}$ \\
\hline $\begin{array}{l}\text { MMP-3 } \\
\text { (Tadbir, AA; 2012) }\end{array}$ & Cases/Controls & $\begin{array}{l}\text { - } 40 \text { OSCC } \\
\text { - } 45 \text { healthy patients }\end{array}$ & ELISA & $\begin{array}{l}\text { - OSCC }=9.45 \pm 4.6 \\
\mathrm{ng} / \mathrm{dl} \\
\text { - } \mathrm{Control}=5.9 \pm 3.6 \\
\mathrm{ng} / \mathrm{dl}\end{array}$ & $\begin{array}{l}\text { Helpful for diagnosis } \\
\text { (not correlated to clinical stages) }\end{array}$ \\
\hline $\begin{array}{l}\text { MMP-9 } \\
\text { (Liu, CJ; 2009) }\end{array}$ & Cases/Controls & - 161 patients & ELISA & $\begin{array}{l}\text { - } \mathrm{LN}(+)=290.22 \pm 28.44 \\
\mathrm{ng} / \mathrm{dl} \\
\text { - } \mathrm{LN}(-)=180 \pm 15.09\end{array}$ & $\begin{array}{l}\text { MMP- } 9>226.7 \mathrm{ng} / \mathrm{ml}=\text { shorter } \\
\text { overall survival }\end{array}$ \\
\hline
\end{tabular}

settled the hyphotesis that if a low $\mathrm{Hb}$ concentration is a predictor of decreased local control, Hb corrections may significantly improve tumor oxygenation and prognosis. They found that anemia was significant for the development of lymph node metastasis as well as for the de- velopment of local recurrence. Preoperative transfusion or erythropoietin administration before surgery has very important economic as well as physiologic consequences so this idea should be considered with caution.

Further investigations are needed in a prospective set- 
Table 2. (continue) Summarize evidence on selected papers.

\begin{tabular}{|c|c|c|c|c|c|}
\hline & & & & $\mathrm{ng} / \mathrm{dl}$ & \\
\hline $\begin{array}{l}\text { Nitric Oxide (Ratajczak-wrona, } \\
\text { W; 2013) }\end{array}$ & Cases/Controls & $\begin{array}{l}24 \text { OSCC } \\
-15 \text { healthy patients }\end{array}$ & ELISA & - Higher in stage IV & $\begin{array}{l}\text { Nitric Oxide concentration } \\
\text { correlated with stage }\end{array}$ \\
\hline $\begin{array}{l}\text { VEGF } \\
\text { (Liu, CJ; 2009) }\end{array}$ & Cases/Controls & - 161 patients & ELISA & Cut off $=497.04 \mathrm{pg} / \mathrm{ml}$ & $\begin{array}{l}\text { VEGF }>497.04 \mathrm{pg} / \mathrm{ml} \\
\text { Shorter overall survival }\end{array}$ \\
\hline $\begin{array}{l}\text { Phosphodiesterase (Prabhu, K; } \\
\text { 2011) }\end{array}$ & Cases/Controls & $\begin{array}{l} \\
\end{array} 20$ OSCCalthy patients & ELISA & $\begin{array}{l}\text { - } \text { OSCC }=63.3 \mu \mathrm{mol} / \mathrm{ml} \\
\text { - } \text { Controls }=12 \mu \mathrm{mol} / \mathrm{ml}\end{array}$ & $\begin{array}{l}\text { PDE levels increased in OSCC. } \\
\text { Correleatedqith advanced stage of } \\
\text { cancer. }\end{array}$ \\
\hline $\begin{array}{l}\text { Placenta Growth Factor } \\
\text { (Cheng, SJ;2012) }\end{array}$ & Cases/Controls & $\begin{array}{l}\text { - } 72 \quad \text { OSCC } \\
\text { (before/after } \\
\text { surgery) } \\
\text { - } 30 \text { healthy patients }\end{array}$ & ELISA & $\begin{array}{l}\text { - OSCC Presurgery= } \\
19.1 \pm 19.7 \mathrm{pg} / \mathrm{ml} \\
\text { - OSCC } \\
\text { Postsurgery= } 11.0 \pm 6.6 \\
\mathrm{pg} / \mathrm{ml} \\
\text { - Controls= } 10.1 \pm 4.5 \\
\mathrm{pg} / \mathrm{ml}\end{array}$ & Shows prognosis, recurrence. \\
\hline $\begin{array}{l}\text { SCCAg } \\
\text { (Feng; 2010) }\end{array}$ & Cases/Controls & $\begin{array}{l}\text { - } 70 \text { OSCC } \\
\text { - } 72 \text { healthy patients }\end{array}$ & ELISA & Cut off $=1.5 \mathrm{ng} / \mathrm{ml}$ & Decrease after surgery \\
\hline $\begin{array}{l}\text { VEGF } \\
\text { (Feng; 2010) }\end{array}$ & Cases/Controls & $\begin{array}{l}\text { - } 70 \text { OSCC } \\
\text { - } 72 \text { healthy patients }\end{array}$ & ELISA & Cut off $=1.7 \mathrm{ng} / \mathrm{ml}$ & $>$ specificity \\
\hline $\begin{array}{l}\text { Cycling D1 } \\
\text { (Feng; 2010) }\end{array}$ & Cases/Controls & $\begin{array}{l}\text { - } 70 \text { OSCC } \\
\text { - } 72 \text { healthy patients }\end{array}$ & ELISA & Cut off $=1.7 \mathrm{ng} / \mathrm{ml}$ & $\begin{array}{l}\text { >sensitivity } \\
\text { >accuracy }\end{array}$ \\
\hline $\begin{array}{l}\text { Serum fucose } \\
\text { (Pajkumar, N; 2011) }\end{array}$ & Cases/Controls & $\begin{array}{l}\text { - } 53 \text { OSCC } \\
\text { - } 14 \text { healthy patients }\end{array}$ & $\begin{array}{l}\text { Cystein Reagent } \\
\text { (Winzler) }\end{array}$ & $\begin{array}{l}\text { - OSCC }=15.34 \pm 0.86 \\
\mathrm{mg} \% \\
\text { - Controls }=5.323 \pm 6.767 \\
\mathrm{mg} \%\end{array}$ & \\
\hline $\begin{array}{l}\text { Serum Leptin } \\
\text { (Harshkant, P;2010) }\end{array}$ & Cases/Controls & $\begin{array}{l}\text { - } 31 \text { OSCC } \\
\text { - } 28 \text { health ypatients }\end{array}$ & ELISA & $\begin{array}{l}-\mathrm{OSCC}=2.76 \pm 1.98 \\
\mathrm{ng} / \mathrm{ml} \\
- \text { Controls }=5.71 \pm 3.58 \\
\mathrm{ng} / \mathrm{ml}\end{array}$ & $\begin{array}{l}\text { Serum leptin could provide state } \\
\text { of cachexia in OSCC PATIENTS }\end{array}$ \\
\hline $\begin{array}{l}\text { Sialic acid levels } \\
\text { (Joshi, M; 2011) }\end{array}$ & Cases/Controls & $\begin{array}{l}\text { - } 30 \text { OSCC } \\
\text { - } 30 \text { oral precancer } \\
\text { - } 30 \text { healthy patients }\end{array}$ & $\begin{array}{l}\text { Resorcinol } \\
\text { Reagent } \\
\text { Method }\end{array}$ & $\begin{array}{l}\text { - } \mathrm{OSCC}= \\
84.44 \pm 8.26 \mathrm{mg} / \mathrm{dl} \\
- \text { Precancer }= \\
66.95 \pm 4.61 \mathrm{mg} / \mathrm{dl} \\
\text { - } \text { Control group = } \\
58.59 \pm 5.81 \mathrm{mg} / \mathrm{dl}\end{array}$ & \\
\hline $\begin{array}{l}\text { Th17 cells } \\
(\mathrm{Li}, \mathrm{C} ; 2011)\end{array}$ & Cases/Controls & $\begin{array}{l}\text { - } 67 \text { HNSCC } \\
\text { - } 21 \text { healthy patients }\end{array}$ & $\begin{array}{l}\text { Flow cytometric } \\
\text { analysis } \\
\text { ELISA }\end{array}$ & $\begin{array}{l}\text { HNSCC }=1.0 \pm 0.4 \% \\
\text { LN }(+)=1.4 \pm 0.6 \% \\
\text { LN( }(-)=0.7 \pm 0.3 \% \\
\text { Controls }=0.3 \pm 0.1 \%\end{array}$ & $\begin{array}{l}\text { TH17 cells proportion increases } \\
\text { in advanced HNSCC }\end{array}$ \\
\hline $\begin{array}{l}\text { TPA } \\
\text { (Sawant, SS; 2011) }\end{array}$ & Cases/Controls & $\begin{array}{l}\text { - } 80 \text { OSCC } \\
\text { - } 24 \text { healthy patients }\end{array}$ & IRMA & Cut off $=140.93 \mathrm{U} / \mathrm{L}$ & Prediction of recurrence \\
\hline $\begin{array}{l}\text { VEGF-A } \\
\text { (Nayak, S; 2011) }\end{array}$ & Cases/Controls & $\begin{array}{l}\text { - } 60 \text { OSCC } \\
\text { - } 60 \text { oral precancer } \\
\text { - } 20 \text { healthy patients }\end{array}$ & $\begin{array}{l}\text { ELISA } \\
\text { PCR }\end{array}$ & $\begin{aligned}- & \text { OSCC }= \\
& 1264.08 \pm 1216.70 \\
\mathrm{pg} / \mathrm{ml} & \\
- & \text { Precancer }= \\
& 462.54 \pm 322.76 \mathrm{pg} / \mathrm{ml} \\
- & \text { Control group }= \\
& 187.91 \pm 106.75 \mathrm{pg} / \mathrm{ml}\end{aligned}$ & VEGF level upregulated in OSCC \\
\hline $\begin{array}{l}\text { VEGF } \\
\text { (Friedrich; 2010) }\end{array}$ & Cases/Controls & - 86 OSCC patients & ELISA & High variability. & $\begin{array}{l}\text { No correlation with clinical } \\
\text { stages }\end{array}$ \\
\hline $\begin{array}{l}\text { Visfatin/pre-b cell colony } \\
\text { enhancing factor (Tsai, YD; } \\
\text { 2013) }\end{array}$ & Cases/Controls & $\begin{array}{l}\text { - } 51 \text { OSCC } \\
\text { - } 57 \text { healthy patients }\end{array}$ & ELISA & $\begin{array}{l}\dot{\mathrm{OSCC}}=7.0 \pm 4.5 \mathrm{ng} / \mathrm{ml} \\
\text { Control }=4.8 \pm 1.9 \mathrm{ng} / \mathrm{ml}\end{array}$ & $\begin{array}{l}\text { Significant correlation with white } \\
\text { blood cell count }\end{array}$ \\
\hline
\end{tabular}

ting, with greater evidence, to rule out dependency with other more important factors.

-Cytokines

Proinflammatory cytokines interleukin 1 beta (IL-1 $\beta$ ), interleukin 6 (IL-6) and tumor necrosis factor alpha $(\mathrm{TNF}-\alpha)$ regulates inflammatory response and play significant role in the development of cancer (29).
In their study, Brailo et al. (30) showed that patients with oral cancer have higher salivary IL- $1 \beta$ and IL-6 concentrations compared to patients with leukoplakia and healthy con-trol but no significant differences in serum IL-6 were observed between the groups. However, serum TNF- $\alpha$ concentration was significantly higher in control subjects compared to oral cancer patients. 
Table 3. Biomarkers identified in studies.

\begin{tabular}{|c|c|c|}
\hline 1. Adiponectine & $\begin{array}{l}\text { Adiponectin is an adipokine produced predominantly by } \\
\text { Adipocytes. It functions as an anti-diabetic, anti- } \\
\text { atherogenic, anti-inflammatory and anti-angiogenic } \\
\text { hormone. }\end{array}$ & Associated biomarker \\
\hline 2. Annexin A1 mRNA & $\begin{array}{l}\text { Annexin A1 an anti-inflammatory and calcium-dependent } \\
\text { protein of the superfamily of annexins, may have important } \\
\text { regulatory roles in tumor development and progression }\end{array}$ & Associated biomarker \\
\hline 3. CRP & $\begin{array}{l}\text { C-Reactive Protein (CRP)is a functional analogue to } \\
\text { immunoglobulin G, which synthesis by pro-inflammatory } \\
\text { cytokines }\end{array}$ & Associated biomarker \\
\hline 4. Cycling D1 & $\begin{array}{l}\text { Cyclin D1, the product of the CCND1 gene located on } \\
\text { chromosome } 11 \mathrm{q} 13\end{array}$ & Associated biomarker \\
\hline 5. DCR3 & $\begin{array}{l}\text { Decoy receptor 3. DcR3 functions as a death decoy } \\
\text { inhibiting apoptosis mediated by the tumor necrosis factor } \\
\text { receptor family. }\end{array}$ & Specific biomarker \\
\hline 6. GDF 15 & $\begin{array}{l}\text { Growth-differentiation factor } 15 \text { (GDF } 15) \text { is involved in } \\
\text { tumor pathogenesis. Its expression is increased in many } \\
\text { types of cancers. (associated biomarker) }\end{array}$ & Specific biomarker \\
\hline 7. $\mathrm{Hb}$ & $\begin{array}{l}\text { Hemoglobine level mediates tumor response to radiation } \\
\text { through the delivery of oxygen tothe tumor. }\end{array}$ & Associated biomarker \\
\hline 8. $\quad$ TNFa & Tumor necrosis factor-alpha & Specific biomarker \\
\hline 9. IL6 & Interleukin 6. Proinflammatory cytokines & Associated biomarker \\
\hline 10. $\mathrm{MiCB}$ & $\begin{array}{l}\text { Major histocompatibility complex class I-related chain A/B } \\
\text { (MICA/B), a ligand of natural killer group 2D (NKG2D) } \\
\text { immunoreceptors. }\end{array}$ & \\
\hline 11. MMP-3 & $\begin{array}{l}\text { Matrix metalloproteinase- } 3 \text { is a member of MMP family } \\
\text { which is capable to degrade a broad range of substrates. } \\
\text { MMP-3 reveals pathological expression in many tumors. }\end{array}$ & Associated biomarker \\
\hline 12. MMP-9 & $\begin{array}{l}\text { Matrix metalloproteinase-9. Potent factors involved in } \\
\text { angiogenesis. Under physiological conditions MMP are } \\
\text { capable of degrading extracellular matrix and basement } \\
\text { membrane components. }\end{array}$ & Associated biomarker \\
\hline 13. Nitric Oxide & $\begin{array}{l}\text { Nitric Oxide concentration plays an essential role in the } \\
\text { process of lipid peroxidation. }\end{array}$ & Associated biomarker \\
\hline 14. PDEs & $\begin{array}{l}\text { Phosphodiesterases have a fundamental role in the } \\
\text { transduction of the intracellular signals and tumor growth } \\
\text { by influencing angiogenesis. }\end{array}$ & Associated biomarker \\
\hline 15. PIGF & $\begin{array}{l}\text { Placenta growth factor is a member of the vascular } \\
\text { endothelial growth factor (VEGF) family.PlGF stimulates } \\
\text { proliferation, differentiation, and survival of endothelial } \\
\text { cells. }\end{array}$ & Associated biomarker \\
\hline 16. SCCAg & $\begin{array}{l}\text { Squamous cell carcinoma antigen. A tumor-associated } \\
\text { protein, } \\
\text { an adjunct in the diagnosis of the disease (associated } \\
\text { biomarker) }\end{array}$ & Specific biomarker \\
\hline 17. Serum fucose & $\begin{array}{l}\text { L-fucose, is a monosaccharides that compounds serum } \\
\text { glycoproteins. }\end{array}$ & Associated biomarker \\
\hline 18. Serum Leptin & $\begin{array}{l}\text { Leptin is a protein of cytokine family, related to body } \\
\text { weight, metabolism and reproductive function }\end{array}$ & Associated biomarker \\
\hline 19. Sialic acid levels & $\begin{array}{l}\text { Siacilic acids are acetylated derivatives of neuramic acid. } \\
\text { They are attached to the non-reduced residue of } \\
\text { carbohydrate chains of glycoproteins and glycolipids. }\end{array}$ & Associated biomarker \\
\hline 20. Th17 cells & $\begin{array}{l}\text { TH17 cells are the third subset of CD4+ T helper cells ( } \mathrm{T} \\
\text { lymphocytes that belong to the CD4+ subset). Important } \\
\text { role in inflammation. }\end{array}$ & Associated biomarker \\
\hline 21. TPA & $\begin{array}{l}\text { Tissue polypeptide Antigen. } \\
\text { TPA is one of the most frequently used cytokine evaluated } \\
\text { as a serum marker }\end{array}$ & Associated biomarker \\
\hline 22. VEGF & $\begin{array}{l}\text { Vascular endothelial growth factor. } \\
\text { VEGF is a multifunctional cytokine that plays a pivotal role } \\
\text { in angiogenesis. (induction of angiogenesis in tumour } \\
\text { growth) }\end{array}$ & Associated biomarker \\
\hline $\begin{array}{l}\text { 23. Visfatin/pre-b cell } \\
\text { colony enhancing } \\
\text { factor }\end{array}$ & $\begin{array}{l}\text { Nicotiamidephosphoribosyltransferase or pre-B cell colony } \\
\text { enhancing factor, is a pro-inflammatory cytokine.It } \\
\text { regulates growth, apoptosis, and angiogenesis. }\end{array}$ & Associated biomarker \\
\hline
\end{tabular}


Chang et al. (16) conducted a study to demonstrate the possible biologic relevance of potential cytokine markers in OSCC. They analyzed the associations between the clinicopathologic manifestations of OSCC and the blood levels of the 12 individual cytokines. As Brailo et $a l$. (30) did before, they find strong associations between some increased cytokine levels and clinical factors but the study did not reveal any associations between others cytokines with elevated levels in OSCC patients and clinicopathologic manifestations.

These investigations fails to identify certain cytokines or cytokine panels that could be used to effectively detect OSCC patients, Results from this studies and heterogeneous literature data indicate that altered cytokine production and responsiveness in oral cancer takes place primarily in the oral cavity and does not reflect on serum cytokine concentrations

-Major complex class I-related chain A/B (MIC-B)

Expression of MIC-A/B, ligands of natural killer group $2 \mathrm{D}$, has been proposed to play an important role in tumor immunosurveillance. Soluble forms of MICA/B are increased in sera of cancer patients and are postulated to impair antitumor immune response by down regulating expression of NKG2D immunoreceptors. In advanced stages of some tumors have been reported increases in soluble MIC-A (31). Watson et al. (31) found that OSCC patients with high soluble MIC-B levels had significantly lower survival rates. Furthermore, patients with both high soluble MIC-A and soluble MIC-B levels also had markedly decreased survival rates.

Tamaki et al. (32) reported that serum MICB levels did not differ significantly from those in normal control individuals. However, they indicated that serum MICB levels were significantly increased in stage IV OSCC and it was significantly associated with decreased survival rates in patients. These findings suggest the utility of sMICB levels as a marker for tumor progression.

-Matrix metalloproteinase enzymes (MMPs)

MMPs are proteolytic enzymes and in cancer they regulate various cell behaviors by degradation of proteins. These include cancer cell growth, differentiation, apoptosis, migration, invasion and regulation of tumor angiogenesis and immune surveillance.

Liu et al. (33) analyzed the association between pretreatment serum levels of MMP-9 and clinic-pathological parameters and outcome for patients with OSCC. In this investigation patients with MMP-9 serum levels higher than median $(226.7 \mathrm{ng} / \mathrm{mL})$ had significantly shorter overall survival than those with levels lower than median. It suggested pretreatment serum levels of MMP-9 as a powerful prognostic marker in patients with oral squamous cell carcinoma.

Tadbir et al. (34), analysed serum MMP-3 level in OSCC patients. Their results showed that serum MMP-3 level in OSCC patients was significantly higher than healthy controls but they couldn't correlate serum MMP-3 concentration with the clinicopathological. Unlike the previously mentioned study, the results suggest that the measurement of serum MMP-3 concentration might be helpful to diagnose OSCC but not to predict prognosis. -Squamous cell carcinoma antigen (SCC-Ag)

SCC-Ag, a tumor-associated protein, was first isolated as "TA-4" from SCC tissue of the uterine cervix in 1977 (35). Since then, several studies have shown that serum SCCA was elevated in OSCC patients and could be used as an adjunct in the diagnosis of the disease. Recently some studies have found that serum SCC-Ag concentrations were significantly increased in OSCC patients, and that the SCC-Ag level decreased significantly after tumor resection (22).

SCC-Ag serum level was also correlated with tumor. Moreover other investigations mentioned it may be a useful tool for monitoring the course of the disease and its recurrence (22).

These studies shows evidence enough to remark the utility of SCC-Ag, a specific antigen, in the diagnosis and prognosis of oral cancer if serum levels are well controlled during preoperative and the follow-up.

-Sialic acid level

Siacilic acids are acetylated derivatives of neuramic acid. They are attached to the non-reduced residue of carbohydrate chains of glycoproteins and glycolipids. Altered glycosylation of glycoconjugates is among the important molecular changes that accompany malignant transformation (35) .

Joshi (12) found the mean serum total sialic acid levels in control group $(58.59 \pm 5.81 \mathrm{mg} / \mathrm{dl})$, oral precancer $(66.95 \pm 4.61 \mathrm{mg} / \mathrm{dl})$ and oral cancer group $(84.44 \pm 8.26 \mathrm{mg} /$ dl) were statistically significant $(p<0,005)$. These differences were also found by Rapjura et al. (36) (control group $=30.25 \pm 2.49 \mathrm{mg} / \mathrm{dl}$; cancer group $=63.70 \pm 19.40 \mathrm{mg} /$ dl). Sialic acid level is directly proportional to tumor burden $(35,36)$. Joshi (12) found the mean serum total sialic acid levels in stage I was $71,24 \mathrm{mg} / \mathrm{dl}$ whereas it was $73,36 \pm 4,65 \mathrm{mg} / \mathrm{dl}, 84,61 \pm 6,40 \mathrm{mg} / \mathrm{dl}$, and $89,34 \pm 4,68$ $\mathrm{mg} / \mathrm{dl}$ in stage II, stage III and stage IV respectively.

\section{$-\mathrm{T}(\mathrm{h}) 17$}

TH17 cells are the third subset of CD4+ Thelper cells (T lymphocytes that belong to the $\mathrm{CD} 4+$ subset), which are characterized by their production of interleukin (IL).

$17 \mathrm{~A}$ and IL-17F have been verified to play an important role in inflammation, autoimmune diseases, and human organ transplantation rejection. Li et al. (37), reported an increase of serum IL-17 levels in patients with head and neck squamous cell carcinomas (HNSCC) compared with healthy control subjects $(123.35-45.13 \mathrm{pg} / \mathrm{mL}$ vs. $20.78-3.95 \mathrm{pg} / \mathrm{mL} ; p<0.05)$. The results indicated that IL-17 expression can be detected in the very early stage of squamous cell carcinoma and increases gradually with the development of the tumor. There was significant difference between TH17 cell proportions in peripheral 
blood in patients with or without lymph node metastasis. This study suggested that TH17 cells may be involved in tumor growth and metastasis of HNSCC.

-Tissue polypeptide Antigen (TPA)

TPA is one of the most frequently used cytokine evaluated as a serum marker for its clinical applications. In their study Sawant et al. (38), using immunoradimetric assay, found that elevated levels of TPA was correlated significantly with stage $(p=0.02)$, development of recurrence $(p<0.006)$, and impacted survival $(p<0.033)$. This result indicates that TPA can be a useful tumor marker for the prediction of recurrence and poor prognosis in human oral cancer.

-Vascular endothelial growth factor (VEGF)

VEGF is multifunctional cytokine that plays a pivotal role in angiogenesis. It has been considered as the most potent one for the induction of angiogenesis in tumor growth. Shang et al. (39), determinate that serum VEGF concentration was increased in patients with OSCC (Control group $=148.80 \pm 64.17 \mathrm{pg} / \mathrm{ml}$, Cancer $=567.97 \pm 338.17 \mathrm{pg} /$ ml. $P<0.001)$ Increased values of VEGF has been found with progression of disease and decreased values after surgery. Higher level of serum VEGF was closely associated with lymph node metastasis 33 and clinical stage in OSCC patients $(33,39)$. Finally, elevated serum VEGF levels have been correlated with poor disease-free survival and poor progression-free survival in cancer patients (33).

We have found quite homogeneous criteria and protocol to investigate the role of serum biomarkers but there is still no unified criteria for using a certain marker or another.

Our results highlight that a wide variety of biomarkers have been studies and a great part of theme have demonstrated their effectiveness in the diagnosis and/or prognosis of oral cancer. Most of the investigations are cases and controls studies where the measurement chosen system is ELISA. Surprisingly, the quality of the articles included was acceptable and were classified as "low risk of bias". The main limitation of the studies in our systematic review is that there is no a real follow-up of the patients and they do not repeat all the measurements in serum. We think this is crucial to correlate biological values with the progression and prognosis of the disease so future investigations should contemplate this item to provide more evidence of the utility of serum biomarkers. Biomarker use for diagnosis and prognosis is supported by clinical and scientific evidence is relevant. Nevertheless, after selecting a certain biomarker, monitoring protocols should be established in oral and maxillofacial surgeons teams so as we have a correct understanding of biological values.

\section{References}

1. Jemal A, Siegel R, Ward E, Hao Y, Xu J, Thun MJ. Cancer statistics, 2009. CA Cancer J Clin. 2009; 59:225-249.

2. Tanaka T, Ishigamori R. Understanding carcinogenesis for fighting oral cancer. J Oncol. 2011;2011:603740.
3. Mishra R. Biomarkers of oral premalignant epithelial lesions for clinical application. Oral Oncol. 2012;48:578-584.

4. Kademani D. Oral cancer. Mayo Clin Proc. 2007;82:878-87.

5. Gupta PC, Murti PR, Bhonsle RB, Mehta FS, Pindborg JJ. Effect of cessation of tobacco use on the incidence of oral mucosal lesions in a 10-yr follow-up study of 12,212 users. Oral Dis. 1995;1:54-8.

6. Bundgaard T, Bentzen SM, Wildt J. The prognostic effect of tobacco and alcohol consumption in intra-oral squamous cell carcinoma. Eur J Cancer B Oral Oncol. 1994;30B:323-8.

7. Sawant SS, Zingde SM, Vaidya MM. Cytokeratin fragments in the serum: Their utility for the management of oral cancer. Oral Oncol. 2008;44:722-32.

8. Bijian K, Mlynarek AM, Balys RL, et al. Serum proteomic approach for the identification of serum biomarkers contributed by oral squamous cell carcinoma and host tissue microenvironment. J Proteome Res. 2009;8:2173-85.

9. Cordes C, Von Lingen J, Gorogh T, Ambrosch P, Gottschlich S, Hoffmann M. Molecular and immunological aspects of p53 and p53autoantibodies in head and neck squamous cell carcinoma. Oncol Rep. 2009;22:1299-303.

10. Kato H, Torigoe T. Radioimmunoassay for tumor antigen of human cervical squamous cell carcinoma. Cancer. 1977;40:1621-8.

11. Hoffmann-Fazel A, Hoffmann M, Gottschlich S, Maass JD, Rudert H, Maune S. Cyfra 21-1 in diagnosis of distant metastases of head and neck carcinoma. Anticancer Res. 2003;23:917-20.

12. Joshi M PR. Estimation and comparative study of serum total sialic acid levels as tumor markers in oral cancer and precancer. J Cancer Res Ther. 2010;6:263-6.

13. Doweck I, Barak M, Uri N, Greenberg E. The prognostic value of the tumour marker cyfra 21-1 in carcinoma of head and neck and its role in early detection of recurrent disease. Br J Cancer. 2000;83:1696-701.

14. Clementini M, Morlupi A, Agrestini C, Barlattani A. Immediate versus delayed positioning of dental implants in guided bone regeneration or onlay graft regenerated areas: A systematic review. Int J Oral Maxillofac Surg. 2013;42:643-50.

15. Chang KP, Wu CC, Fang KH, et al. Serum levels of chemokine (C-X-C motif) ligand 9 (CXCL9) are associated with tumor progression and treatment outcome in patients with oral cavity squamous cell carcinoma. Oral Oncol. 2013;49:802-7.

16. Chang KP, Chang YT, Liao CT, et al. Prognostic cytokine markers in peripheral blood for oral cavity squamous cell carcinoma identified by multiplexed immunobead-based profiling. Clin Chim Acta. 2011;412:980-7.

17. Inal E, Lacin M, Asal K, et al. The significance of ferritin, lipidassociated sialic acid, CEA, squamous cell carcinoma (SCC) antigen, and CYFRA 21-1 levels in SCC of the head and neck. Kulak Burun Bogaz Ihtis Derg. 2004;12:23-30.

18. Madsen EL, Rissanen A, Bruun JM, et al. Weight loss larger than $10 \%$ is needed for general improvement of levels of circulating adiponectin and markers of inflammation in obese subjects: A 3-year weight loss study. Eur J Endocrinol. 2008;158:179-87.

19. Guo XH, Wang JY, Gao Y, et al. Decreased adiponectin level is associated with aggressive phenotype of tongue squamous cell carcinoma. Cancer Sci. 2013;104:206-13.

20. Perretti M, Flower RJ. Annexin 1 and the biology of the neutrophil. J Leukoc Biol. 2004;76:25-9.

21. Faria PC, Sena AA, Nascimento R, et al. Expression of annexin A1 mRNA in peripheral blood from oral squamous cell carcinoma patients. Oral Oncol. 2010;46:25-30.

22. Feng XY, Li JH, Li JZ, Han ZX, Xing RD. Serum SCCA, cyfra 21-1, EGFR and cyclin D1 levels in patients with oral squamous cell carcinoma. Int J Biol Markers. 2010;25:93-8.

23. Capaccio P, Pruneri G, Carboni N, Pagliari AV, Quatela M, Cesana BM, Pignataro L. Cyclin D1 expression is predictive of occult metastases in head and neck cancer patients with clinically negative cervical lymph nodes. Head Neck. 2000;22:234-40.

24. Khandavilli SD, Ceallaigh PO, Lloyd CJ, Whitaker R. Serum Creactive protein as a prognostic indicator in patients with oral squamous cell carcinoma. Oral Oncol. 2009;45:912-4. 
25. Tu HF, Liu CJ, Liu SY, Chen YP, Yu EH, Lin SC, Chang KW. Serum decoy receptor 3 level: A predictive marker for nodal metastasis and survival among oral cavity cancer patients. Head Neck. 2011;33:396-402.

26. Zhang L, Yang X, Pan HY, et al. Expression of growth differentiation factor 15 is positively correlated with histopathological malignant grade and in vitro cell proliferation in oral squamous cell carcinoma. Oral Oncol. 2009;45:627-32.

27. Schiegnitz E, Kammerer PW, Koch FP, Kruger M, Berres M, AlNawas B. GDF 15 as an anti-apoptotic, diagnostic and prognostic marker in oral squamous cell carcinoma. Oral Oncol. 2012;48:608-14.

28. Cordella C, Luebbers HT, Rivelli V, Gratz KW, Kruse AL. An evaluation of the preoperative hemoglobin level as a prognostic factor for oral squamous cell carcinoma. Head Neck Oncol. 2011;3:35.

29. Dinarello CA. Biologic basis for interleukin-1 in disease. Blood. 1996;87:2095-2147.

30. Brailo V, Vucicevic-Boras V, Lukac J, et al. Salivary and serum interleukin 1 beta, interleukin 6 and tumor necrosis factor alpha in patients with leukoplakia and oral cancer. Med Oral Patol Oral Cir Bucal. 2012;17:e10-5

31. Watson NF, Spendlove I, Madjd Z, et al. Expression of the stressrelated MHC class I chain-related protein MICA is an indicator of good prognosis in colorectal cancer patients. Int J Cancer. 2006;118:14451452.

32. Tamaki S, Kawakami M, Ishitani A, Kawashima W, Kasuda S, Yamanaka Y, Shimomura H, Imai Y, Nakagawa Y, Hatake K, Kirita T. Soluble MICB serum levels correlate with disease stage and survival rate in patients with oral squamous cell carcinoma. Anticancer Res. 2010;30:4097-101.

33. Liu CJ, Chang KW, Lin SC, Cheng HW. Presurgical serum levels of matrix metalloproteinase-9 and vascular endothelial growth factor in oral squamous cell carcinoma. Oral Oncol. 2009:45:920-5.

34. Tadbir AA, Purshahidi S, Ebrahimi H, et al. Serum level of MMP-3 in patients with oral squamous cell carcinoma--lack of association with clinico-pathological features. Asian Pac J Cancer Prev. 2012;13:4545-8.

35. Rao VR, Krishnamoorthy L, Kumaraswamy SV, Ramaswamy G.Circulating levels in serum of total sialic acid, lipid-associated sialic acid, and fucose in precancerous lesion and cancer of the oral cavity. Cancer Detect Prev. 1998;22:237-40.

36. Rajpura KB, Patel PS, Chawda JG, Shah RM. Clinical significance of total and lipid bound sialic acid levels in oral pre-cancerous conditions and oral cancer. J Oral Pathol Med. 2005;34:263-7.

37. Li C, Zhao Y, Zhang W, Zhang W. Increased prevalence of T(H)17 cells in the peripheral blood of patients with head and neck squamous cell carcinoma. Oral Surg Oral Med Oral Pathol Oral Radiol Endod. 201;112:81-9.

38. Sawant SS, Chaukar DA, Joshi SS, Dange PP, Kannan S, Kane S, et al. Prognostic value of tissue polypeptide antigen in oral squamous cell carcinoma. Oral Oncol. 2011;47:114-120.

39. Shang ZJ, Li JR, Li ZB. Circulating levels of vascular endothelial growth factor in patients with oral squamous cell carcinoma. Int J Oral Maxillofac Surg. 2002;31:495-8. 